\title{
Estética dentária na Atenção Básica em Saúde
}

\author{
Dental aesthetics in Basic Health Care
}

Ritchely Corrêa Ribeiro*
Fábio Garcia Lima**
Tania Izabel Bighetti**

\section{Resumo}

Objetivo: mostrar a viabilidade de se realizar procedimentos estéticos em usuários do Sistema Único de Saúde (SUS). Relato de caso: este trabalho apresenta o relato de um caso em que se realizou uma restauração estética, com o uso de cimento de ionômero de vidro e resina composta, em uma Unidade Básica de Saúde (UBS) da cidade de Pelotas, RS. A paciente expressava demasiado descontentamento estético. No Brasil, a Atenção Básica em Saúde (ABS) é definida como um conjunto de ações, individuais e coletivas, que incluem promoção e proteção da saúde, prevenção de doenças, diagnóstico, tratamento, reabilitação, redução de danos e manutenção da saúde. Para seguir as diretrizes propostas pelo Ministério da Saúde para a ABS, tornam- se imprescindíveis, em cada território, aproximar-se das pessoas e tentar conhecê-las: suas condições de vida, as representações e as concepções que têm acerca de sua saúde. Dessa forma, em Odontologia, a estética do sorriso pode ser considerada como objeto da ABS. O sorriso é tido como ponto de grande importância para a estética e a harmonia facial e justamente por isso acaba sendo um grande problema na vida de muitos indivíduos, influenciando sua autoestima e relações pessoais e profissionais. O SUS tem como diretriz o cuidado voltado na pessoa e não em suas enfermidades. Considerações finais: as restaurações de natureza estética, consideradas procedimentos de maior complexidade, podem ser confeccionadas em UBS, desde que a técnica adequada, durante o procedimento, seja realizada.

Palavras-chave: Atenção básica. Estética dentária. Unidade básica de saúde.

\section{Introdução}

No Brasil, a Atenção Básica em Saúde (ABS) é definida como um conjunto de ações, individuais e coletivas, que incluem promoção e proteção da saúde, prevenção de doenças, diagnóstico, tratamento, reabilitação, redução de danos e manutenção da saúde. ${ }^{1}$ Apesar da importância conferida à ABS nos documentos normativos do Sistema Único de Saúde (SUS), existe uma série de tensões entre uma visão abrangente de atenção básica, segundo a qual a unidade básica de saúde (UBS) deve ser reconhecida como coordenadora do cuidado, responsável pela atenção integral e humanizada dos indivíduos ao longo do tempo, ${ }^{2}$ e uma visão restrita, que entende atenção básica apenas como um pacote mínimo de serviços, focado nas doenças mais prevalentes. ${ }^{2}$

Especificamente em relação à saúde bucal, às tensões relacionadas às diferentes visões sobre a ABS adicionam-se dificuldades oriundas do próprio modelo hegemônico de atenção à saúde bucal privatista. ${ }^{3}$ Juntem-se a isso o escasso interesse do Estado nessa área durante muitas décadas e a consequente inclusão tardia dos profissionais de saúde bucal no Programa Saúde da Família (PSF), ${ }^{3}$ apenas seis anos após o início da implantação desse programa no país. ${ }^{3}$ Apenas em 2004, as questões referentes à saúde bucal tomaram maior relevância, tendo sido lançada a política nacional de saúde bucal (PNSB) Brasil Sorridente. ${ }^{4}$ Foi criada para reorganizar o trabalho em saúde bucal nos três níveis de atenção, destacando "o cuidado como eixo de reorientação do modelo, a humanização do processo de trabalho, a corresponsabilização dos serviços e o desenvolvimento de ações voltadas para as linhas do cuidado e condições especiais de vida". ${ }^{5}$

De modo geral, quando se pensa em clínica, imagina-se um médico prescrevendo um remédio

Acadêmica de Odontologia, Universidade Federal de Pelotas, Pelotas, RS, Brasil.

Doutor em Odontologia (Dentística), professora adjunto III, Faculdade de Odontologia, Universidade Federal de Pelotas, Pelotas, RS, Brasil.

Doutora em Saúde Pública, professora associada II, Faculdade de Odontologia de Pelotas, Universidade Federal de Pelotas, Pelotas, RS, Brasil. 
ou solicitando um exame para comprovar ou não a hipótese de determinada doença. No entanto, a clínica precisa ser muito mais do que isso. ${ }^{6}$ É certo que o diagnóstico de uma doença sempre parte de um princípio universalizante, generalizável para todos, ou seja, ele supõe alguma regularidade e produz uma igualdade. Mas essa universalidade é verdadeira apenas em parte. Isso pode levar à suposição de que sempre bastaria o diagnóstico para definir todo o tratamento para aquela pessoa. Entretanto, como já dizia um velho ditado, "Cada caso é um caso", e esta consideração pode mudar, ao menos em parte, a conduta dos profissionais de saúde. Por exemplo: um paciente apresenta sintomas de depressão, problemas sociais e baixa autoestima e tem seu tratamento psicológico iniciado. Apenas o tratamento com profissionais psicólogos e/ou psiquiatras é suficiente para a recuperação da saúde desse indivíduo? E o quão importante sua estética pode ser considerada para a manutenção de sua saúde? Contudo, tornam-se imprescindíveis, em cada território, aproximar-se das pessoas e tentar conhecê-las: suas condições de vida, as representações e as concepções que têm acerca de sua saúde. ${ }^{5}$ Dessa forma, em Odontologia, a estética do sorriso de um determinado paciente, detentor de características peculiares, pode ser considerada objeto da ABS.

Além disso, quanto às atribuições do cirurgião-dentista na atenção básica, o que se encontra em documentos normatizados pelo Ministério da Saúde é: realizar procedimentos clínicos em saúde bucal, incluindo atendimento das urgências e pequenas cirurgias ambulatoriais; e, quando houver necessidade de procedimentos mais complexos, encaminhar e orientar usuários a outros níveis de assistência, mantendo sua responsabilização pelo acompanhamento do usuário e o segmento do tratamento. Com isso, o cirurgião-dentista pode optar pela realização ou pelo encaminhamento de procedimentos clínicos, ao se julgar ou não capaz da execução.

O objetivo deste estudo é descrever uma estratégia de aproximação da Odontologia estética com a necessidade real de um usuário de uma UBS do município de Pelotas, RS, por meio do relato de um caso clínico, demonstrando a possibilidade de realização de procedimentos de maior complexidade dentro da ABS do SUS.

\section{Relato de caso}

A paciente SROG, do sexo feminino, com 50 anos de idade, residia na cidade de Pelotas, $\mathrm{RS}$, era cadastrada na UBS Arco Íris e já havia sido atendida pela cirurgiã-dentista (CD), para realização de procedimentos restauradores em dentes anteriores com cavidades de cárie.

Todos os procedimentos executados para a resolução do caso foram realizados na UBS Arco Íris. O caso foi avaliado por dois dos autores (FGL e
RCR), por meio de anamnese e exame clínico. Pela anamnese, pôde-se constatar que a paciente estava bastante descontente com sua estética dentária, relatando dificuldade em se relacionar (problemas sociais e psicológicos). Já ao exame clínico, observou-se a presença de uma coloração clara na maioria dos elementos dentários anteriores, comparada à cor A2 da escala Vita. No entanto, o elemento 11, com tratamento endodôntico realizado há mais de cinco anos, possuía uma coloração bastante amarelada, comparada à cor A4 da escala Vita, gerando um contraste bastante nítido no sorriso da paciente (Figura 1).

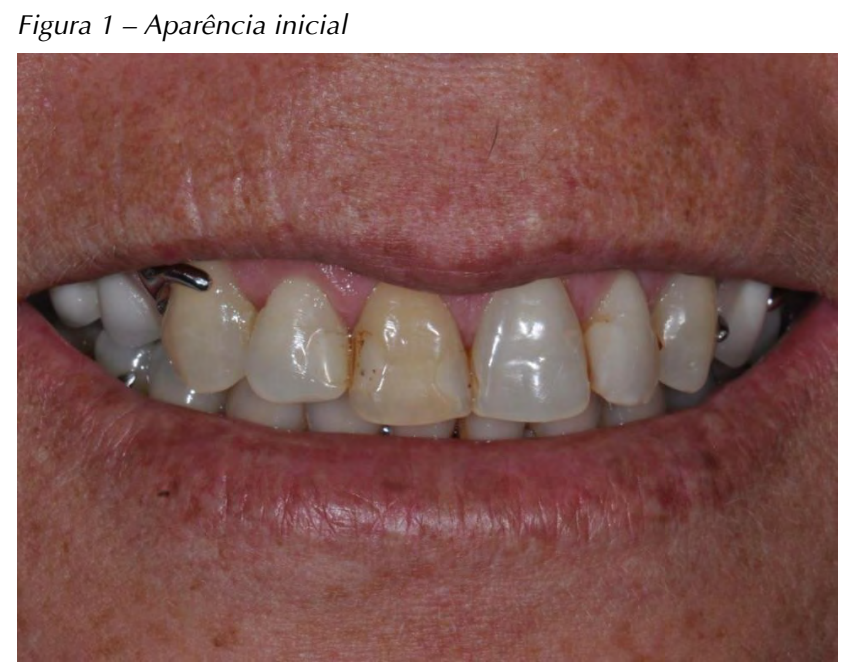

Fonte: autores.

Definida a possibilidade de participação no estudo, a paciente recebeu todas as explicações sobre o tipo de tratamento a ser realizado, suas implicações e os cuidados posteriores, e concordou por meio da assinatura de um termo de consentimento livre e esclarecido.

Antes de os procedimentos restauradores serem executados, foram checadas as guias de desoclusão por movimentos de protrusão (guiados por incisivos inferiores) e lateralidade (guiados por caninos de ambos os lados). Pôde-se concluir que a paciente não possuía contatos prematuros.

O preparo cavitário foi realizado pela técnica da silhueta. ${ }^{7} \mathrm{O}$ desgaste foi iniciado pela região cervical da coroa dentária, com uma ponta diamantada esférica (1014) contornando toda a margem cervical (Figura 2). 
Figura 2 - Desgaste cervical

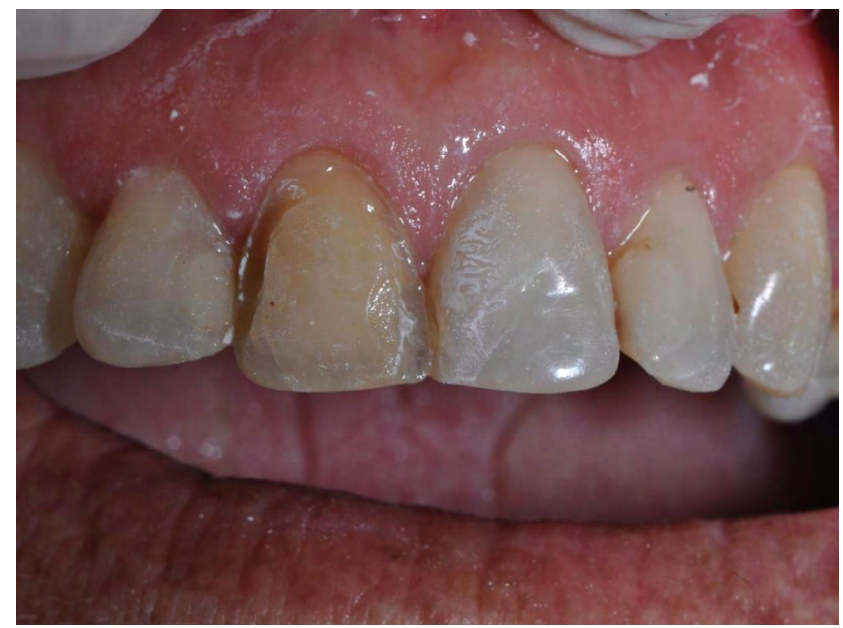

Fonte: autores.

Posteriormente, foi realizada a confecção de dois sulcos de orientações na face vestibular da coroa (Figura 3), com uma ponta diamantada cilíndrica (3215), respeitando as três inclinações existentes na face vestibular de dentes ântero-superiores. Foi averiguado se a profundidade de desgaste dos sulcos estava adequada (cerca de 1,2 $\mathrm{mm}$ de profundidade). Ao obter a profundidade de desgaste desejada, os sulcos foram unidos, para se chegar a um desgaste plano em toda a face vestibular, sempre respeitando as inclinações da face vestibular.

Figura 3 - Desgaste da face vestibular

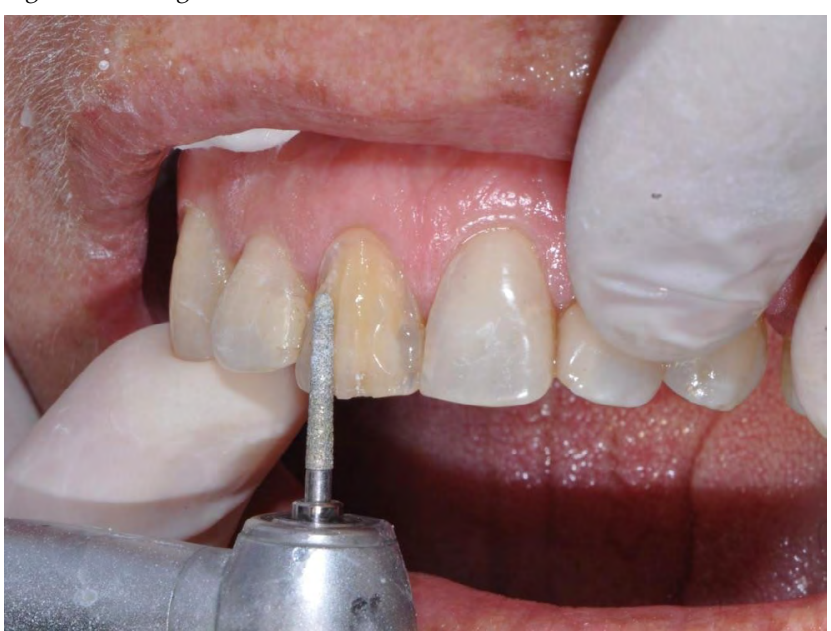

Fonte: autores

Por fim, um desgaste de aproximadamente 1,2 $\mathrm{mm}$ foi realizado na borda incisal da coroa, utilizando também a ponta diamantada cilíndrica (3215). Para tanto, foram feitos dois desgastes de orientação, que foram posteriormente aplainados, finalizando, assim, todo o preparo (Figura 4).
Figura 4 - Redução da borda incisal

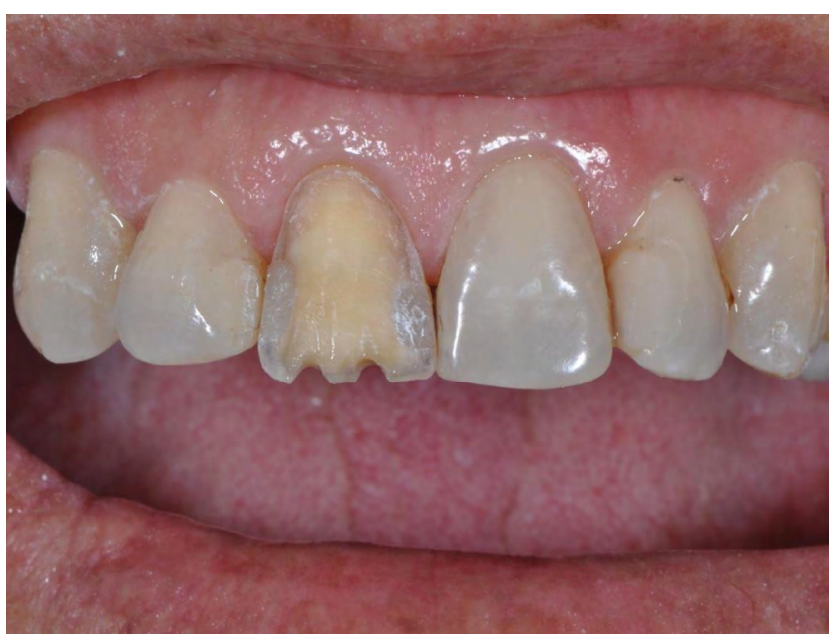

Fonte: autores.

O procedimento restaurador foi iniciado pela restauração da região incisal do dente (Figura 5). Para isso, foi preparado o isolamento do campo com roletes de algodão, e foi inserida uma matriz de poliéster nas proximais do dente, isolando-o de seus adjacentes. Então, o condicionamento da superfície de dentina da região incisal (tanto por vestibular como por palatina) foi realizado com ácido fosfórico a $37 \%$, por 15 segundos. Após enxágue e secagem do substrato, aplicou-se o sistema adesivo Ybond Mono (Yller), com auxílio de um aplicador do tipo microbrush. A secagem e a fotoativação do sistema adesivo foram realizadas conforme as orientações do fabricante. Logo, foi adicionado um incremento de resina FIll Magic(Coltene), de esmalte cor A2, para reconstruir a porção incisal do dente.

Figura 5 - Reconstrução da borda incisal

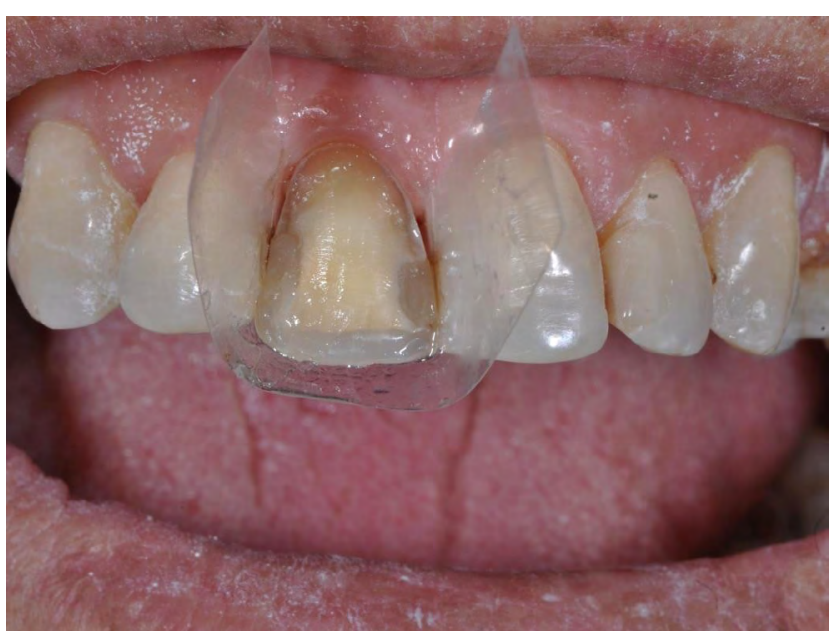

Fonte: autores. 
Após a reconstrução da porção incisal do dente, foi aplicado cimento de ionômero de vidro (CIV) restaurador nos terços médio e cervical do elemento (Figura 6), utilizado como agente de opacificação para a restauração. O CIV substituiu uma camada de resina de dentina com efeito de opacificação, que poderia ter sido utilizada, caso houvesse disponibilidade.

Figura 6-CIV aplicado nos terços cervical e médio

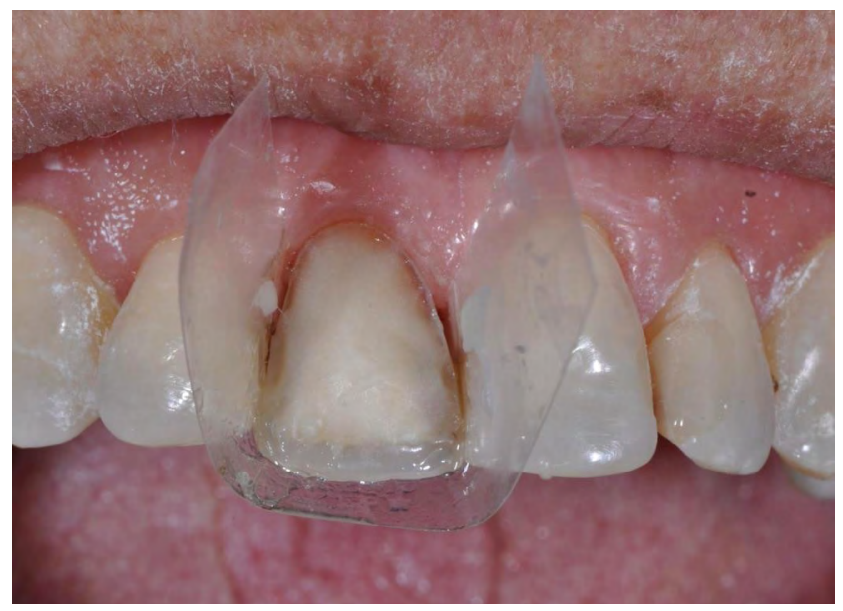

Fonte: autores.

Posteriormente, realizou-se o condicionamento ácido, com ácido fosfórico a $37 \%$, das áreas da face em que não se tinha aplicado o CIV (bordas proximais). E, após lavagem e secagem, o sistema adesivo Mono (Yller) foi aplicado em toda a superfície vestibular com auxílio de um aplicador do tipo $m i$ crobrush (Figura 7). A secagem e a polimerização do sistema adesivo foram feitas conforme recomendações do fabricante. Em seguida, uma camada de resina Fill Magic (Coletene) A2E foi aplicada sobre a superfície até então construída.

A paciente concordou com o tratamento descrito por meio da assinatura de um termo de consentimento livre e esclarecido.

Figura 7 - Aplicação de sistema adesivo para reconstrução da face vestibular

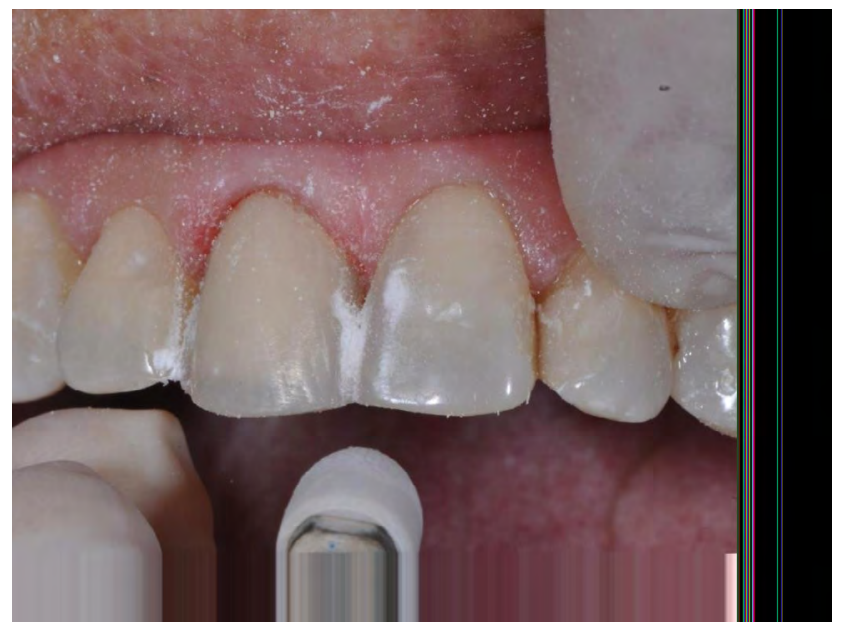

Fonte: autores.
Os desgastes para acabamento da restauração foram realizados com uma ponta diamantada cilíndrica (2135). E o polimento foi realizado com uma ponta para acabamento em formato de chama (Optimize - TDV), em baixa rotação (Figura 8), chegando, com isso, ao resultado final (Figura 9).

Figura 8 - Polimento da restauração

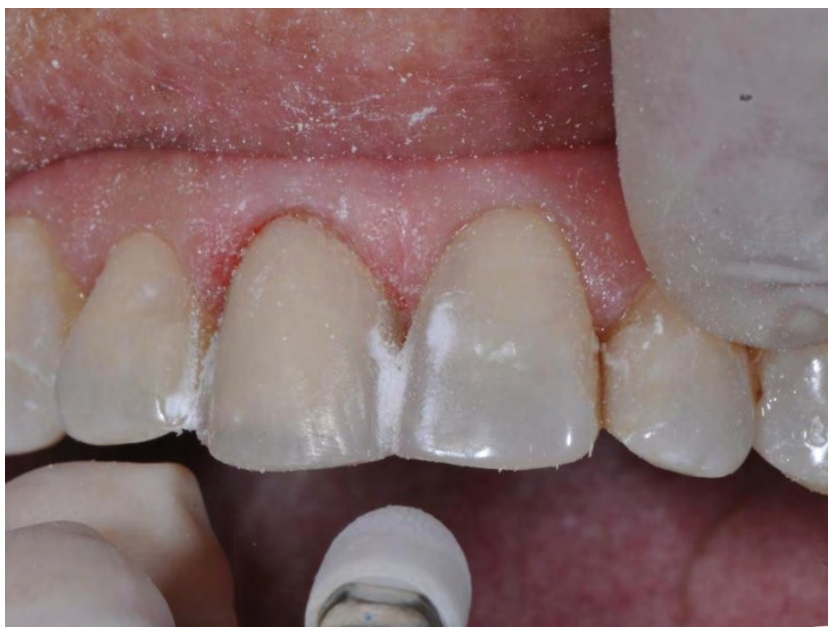

Fonte: autores.

Figura 9 - Aparência final

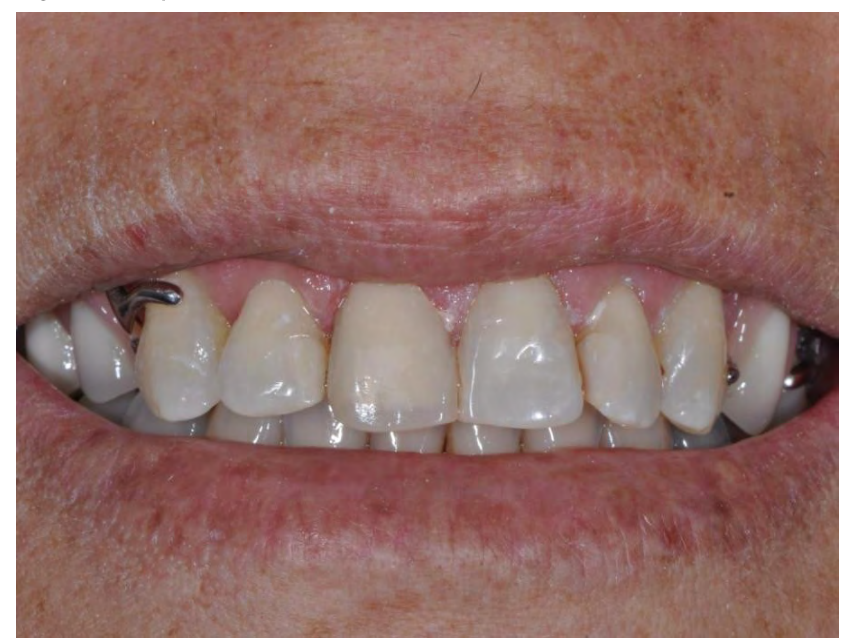

Fonte: autores.

Após a conclusão da restauração, os guias de desoclusão foram novamente checados. Foi necessário fazer um pequeno ajuste, diminuindo a altura cervicoincisal do dente, pois, nos primeiros testes, houve um pequeno ponto de contato prematuro. Ao final, contatos prematuros não foram mais observados, e o dente restaurado passou a ter sua cor mais semelhante à de seus elementos adjacentes.

\section{Discussão}

Autoestima pode ser descrita como uma forma de o ser humano se autoaceitar. Ela é formada por meio de relacionamentos pessoais que se têm desde a infância até a fase adulta e define a percepção que uma pessoa tem de si mesma, ou seja, o quanto ela 
se gosta. Está ligada diretamente à autoconfiança e reflete a forma como a sociedade vê e aceita cada indivíduo. ${ }^{8}$ Entende-se boa autoestima como bem-estar, felicidade e uma avaliação positiva de si. São sensações subjetivas, relacionadas à psicologia, o que faz com que a medicina considere o resultado da intervenção como reparação psicológica. ${ }^{9} \mathrm{Na}$ atualidade, os bem-estares físico e mental são vistos como fatores determinantes para a melhora da autoestima e podem ser encontrados por meio de tratamentos estéticos. ${ }^{10}$

Torna-se perceptível que dar atenção para demandas estéticas de determinados indivíduos deve ser considerado como parte da avaliação geral de sua saúde. E, ainda, tratamentos estéticos podem se fazer necessários para a manutenção das saúdes geral e psicológica de determinado ser. Em Odontologia, tratamentos estéticos têm se tornado rotineiros devido ao crescente interesse dos pacientes por procedimentos que influenciam sua autoimagem, a vaidade e a aceitação social. ${ }^{11}$

A integralidade, proposta pelo SUS, sugere a ampliação e o desenvolvimento do cuidar por parte dos profissionais da saúde. A integralidade pode ser não apenas definida como uma diretriz básica do SUS, mas também percebida como uma visão abrangente dos seres humanos, tratando-os não somente como seres doentes, mas também como pessoas dotadas de sentimentos, desejos e aflições. ${ }^{12}$. Além disso, a PNSB, criada em 2004, propôs o cuidado como eixo de reorientação do modelo de trabalho, a corresponsabilização dos serviços, o desenvolvimento de ações voltadas para as linhas do cuidado e condições especiais de vida e a humanização do processo. ${ }^{5} \mathrm{E}$ o atendimento humanizado pressupõe que, para que se realize uma clínica adequada, é preciso saber, além do que o sujeito apresenta de "igual", o que ele apresenta de "diferente", de singular em relação aos demais, inclusive sinais e sintomas que somente nele se expressam de determinado modo. ${ }^{6}$ Sendo assim, em alguns casos, abordagens estéticas podem e devem ser consideradas como alvos do SUS.

No caso descrito neste estudo, foi realizada uma restauração do tipo faceta, com a utilização de resina composta, para fazer o mascaramento da dentina escurecida no elemento 11 , uma restauração estética. Entretanto, as facetas diretas de resina composta não devem ser utilizadas de forma indiscriminada. Muitas vezes, sugere-se a realização de clareamento dental pelo conservadorismo, pelo baixo custo e pela vasta evidência científica sobre a efetividade nos resultados. No entanto, essa abordagem não poderia ser realizada em uma UBS, pela falta de material clareador disponível nas unidades. Sendo assim, a faceta passou a ser a abordagem escolhida para este caso. Devido às melhorias nas propriedades dos materiais restauradores adesivos, a indicação do uso das resinas compostas passou a ser cada vez mais ampla, e os procedimentos clínicos, mais conservadores. ${ }^{11}$

A ausência de maior variedade de cores e marcas de compósitos de resina mais estéticos poderia ser motivo para o questionamento da eficiência desse tipo de restaurações em UBS. No entanto, o que se propõe é justamente demonstrar que, apesar da disponibilidade restrita de marcas e cores de materiais restauradores, a técnica bem executada pelo profissional CD pode obter um ótimo resultado. O que pôde ser concretizado, pois, ao final do procedimento restaurador, observaram-se obtenção de excelente estética e satisfação da paciente. Desse modo, o conhecimento científico adequado aliado às diretrizes que regem a Odontologia confere ao profissional grandes possibilidades de devolver forma, cor e textura ao dente afetado. Isso demonstra a importância da capacitação do CD para realizar o procedimento, o que muitas instituições de graduação em Odontologia fornecem aos seus acadêmicos. No entanto, alguns CD formam-se sem o devido conhecimento. $\mathrm{E}$ a responsabilidade no tratamento em acompanhar o caso (manutenção) também deve ser atribuída ao CD.

Um dos maiores requisitos para qualquer material restaurador indicado para uso em dentes anteriores é a capacidade de mimetizar os tecidos arredores e não ser facilmente detectado. Os fatores que controlam tal capacidade são a cor e a translucidez do material restaurador. $\mathrm{O}$ material restaurador mais utilizado para mascarar o escurecimento de dentina é a resina, sendo as resinas de efeito opaco as que melhor reproduzem esse mascaramento. No entanto, as resinas que são disponibilizadas às UBS do SUS não são resinas com desempenho estético adequado para reproduzir o mascaramento. Para tanto, foi utilizado o CIV Restaurador Maxxion $\mathrm{R}$ (FGM). Nos CIV, a cor é produzida pelo vidro e por pigmentos selecionados, como óxido férrico ou carbono preto. Ao passo que a cor não representa um grande problema, a translucidez desses materiais pode ser considerada inadequada para algumas finalidades. Os cimentos apresentam uma aparência opaca, e isso pode limitar sua utilização. ${ }^{13}$ Entretanto, para conseguir o mascaramento de uma dentina escurecida, é justamente a opacidade que se busca em um material, o que justifica o uso desse material para o caso apresentado.

Contudo, resolver esse caso de estética odontológica em uma UBS, que afetava a paciente de uma forma ampla, pode levar novas ideias aos CD que realizarem a leitura deste trabalho. Os serviços ofertados pelo SUS na ABS, em Odontologia, poderão ter resolutividade, complexidade e competência ampliadas. Além disso, os pacientes detentores de características semelhantes às demonstradas no caso descrito (dependentes de serviços do SUS, altamente descontentes com a estética de seu sorriso e com consequentes problemas sociais e psicológicos) terão acesso à resolução de um problema que afeta sua saúde geral, o que pode impactar positivamente em suas vidas. E, dessa forma, o cuidado estará voltado ao indivíduo como um todo. 


\section{Considerações finais}

Com o desenvolvimento deste trabalho, foi possível ultimar que restaurações de natureza estética, consideradas procedimentos de maior complexidade, podem ser confeccionadas até mesmo em uma UBS ou em clínicas sem disponibilidade de variedades de materiais odontológicos, desde que a técnica adequada, durante o procedimento, seja realizada. Afirmado isso, coloca-se esse tipo de abordagem ao alcance do CD da ABS, desde que o profissional tenha conhecimentos técnico e científico para tanto. E desmistifica-se a impossibilidade de execução, quando justificada apenas na ausência de determinadas cores ou marcas de materiais.

\section{Abstract}

Objective: to show the feasibility of performing aesthetic procedures in SUS users. Case Report: This paper reports on a case where an aesthetic restoration was carried out, using glass ionomer cement and composite resin, inside a Basic Health Unit in the city of Pelotas, RS. The patient expressed too much aesthetic discontent. In Brazil, Basic Health Care is defined as a set of individual and collective actions, which include promotion and protection of health, disease prevention, diagnosis, treatment, rehabilitation, harm reduction and health maintenance. To follow the guidelines proposed by the Ministry of Health to the Basic Health Care, it is essential in each territory to approach people and try to know them: their living conditions, representations and conceptions about their health. Thus, in Odontology, the aesthetics of the smile can be considered as Basic Health Care object. The smile is considered as a point of great importance for aesthetics and facial harmony and precisely for this reason it ends up being a great problem in the life of many individuals, influencing their self- esteem and personal and professional relationships The Unified Health System has as guideline the care focused on the person and not on their infirmities. Final considerations: restorations of aesthetic nature, considered as procedures of greater complexity, can be made in UBS, provided that the appropriate technique, during the procedure, is performed.

Keywords: Primary care. Dental aesthetics. Basic health unit.

\section{Referências}

1. Brasil. Política Nacional de Atenção Básica [Internet]. Vol. I, Ministério da Saúde. 2012. 110 p. Available from: URL: http://189.28.128.100/dab/docs/publicacoes/geral/pnab.pdf

2. Starfield B, Shi L. Policy relevant determinants of health: an international perspective. Health Policy (New York) 2002; 60(3):201-18

3. Narvai PC, Frazão P. Saúde bucal no Brasil: muito além do céu da boca. Rio de Janeiro: Fiocruz, 2008. (Coleção Temas em Saúde).

4. Chaves SCL, Cruz DN, Barros SG, Figueiredo AL. Avaliação da oferta e utilização de especialidades odontológicas em serviços públicos de atenção secundária na Bahia, Brasil. Cad Saúde Pública 2011; 27(1):143-54.

5. Brasil. Ministério da Saúde. Secretaria de Atenção à Saúde. Departamento de Atenção Básica. Diretrizes da política nacional de saúde bucal. Portal da Saúde [Internet]. 2004; 16. Available from: URL: http://189.28.128.100/dab/docs/publicacoes/geral/diretrizes_da_politica_nacional_de_saude_bucal. pdf

6. Brasil. Ministério da Saúde. Secretaria de Atenção à Saúde. Política Nacional de Humanização da Atenção e Gestão do SUS. PNH - Clínica ampliada e compartilhada. 2009. 64 p.

7. Araújo F. Técnica da Silhueta: modificação. Universidade Federal de Minas Gerais; 2011.

8. Guenther ZC. Educando o ser humano - uma abordagem da Psicologia Humanista [Internet]. 1997 [cited 2018 Jun 10]. Available from: URL: http://www.psicopedagogiaonline.com. br/index.php/443-resenha-educando-o-ser- humano-uma-abordagem-da-psicologia-humanista

9. Neto PP, Caponi SNC. A medicalização da beleza. Interface Commun Heal Educ 2007; 11(23):569-84.

10. Borba TJ, Thieves FM. Uma reflexão sobre a influência da estética na auto estima, auto-motivação e bem-estar do ser humano. [Internet] 2011. Available from: URL: http://siaibib01.univali.br/pdf/Tamila\%20Josiane\%20Borba.pdf

11. Araújo ÉP, Rocha Filho LA, Brum GT, Caldo-Teixeira AS. Fechamento de diastemas com restaurações diretas de resina composta - relato de caso clínico. Rev G\&S 2009; 1(3):33-8.

12. Pinho IC, Siqueira JCBA, Pinho LMO. As percepções do enfermeiro acerca da integralidade da assistência. Rev Eletrônica Enferm 2006; 08:42-51.

13. Hickel RA. Various Forms of Glass Ionomers and Compomers. Operative Dentistry Supplement 2001; 26:177-90.

\section{Endereço para correspondência:}

Fábio Garcia Lima

Rua Gonçalves Chaves, 457, sala 207

96090-560, Pelotas, RS, Brasil

Telefone: (+55) (53) 9811-4003

E-mail: limafg@hotmail.com 